Military Technical College

Kobry El-Kobbah, Cairo, Egypt

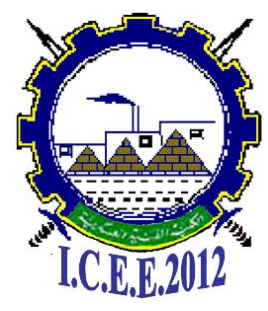

$6^{\text {th }}$ International Conference

on

Chemical \& Environmental

Engineering

29 -31 May, 2012.

CPTB-4

\title{
SYNTHESIS OF POLYANILINE/NIZN-FERRITE NANO-STRUCTURED COMPOSITE AND ITS MICROWAVE ABSORPTION CHARACTERISTICS IN 8-18GHZ
}

\author{
Khaled Aly ${ }^{*}$, Fayed M.S*, M.K. Abd El Magid T* and Ayman El Tager*
}

\begin{abstract}
.
Core-shell composite structure of NiZn-ferrite coated with polyaniline was prepared by in situ polymerization of aniline at different aniline-volume to ferrite-weight ratios (Aniline/NiZnferrite $=1 / 1,2 / 1,3 / 1)$. The composite samples were introduced into epoxy resin forming sheet to serve as a microwave absorber. Chemically and morphologically, the composite samples were characterized by FTIR, XRD, EDAX and TEM. The electrical conductivity of the composites was measured by four-point probe technique. Microwave absorbing performances were investigated by measuring reflectivity in $8-18 \mathrm{GHz}$ using PR-17 microwave reflectometer. IR analysis indicated successful formation of polyaniline. XRD revealed a spinel structure of the prepared NiZn-ferrite and EDAX indicated the right elemental composition of the produced ferrite. TEM clearly confirmed the core-shell structure of prepared NiZn-ferrite/polyaniline composite. It is found that the electrical conductivity of the NiZn-ferrite particles can be enhanced by the conductive PANI coating and increased by increasing the PANI/ferrite ratio in the composite. All samples were found to absorb microwave with some differences in the average reflection loss with respect to preparation ratios. In general, the maximum reflection loss frequency can be determined by controlling aniline to NiZn-ferrite ratio.
\end{abstract}

Keywords: Microwave absorbing materials; NiZn-ferrite; polyaniline; reflection loss; reflectometer.

\footnotetext{
${ }^{*}$ Egyptian Armed Forces
} 


\section{Introduction}

Microwaves are electromagnetic radiation with frequencies ranging between $0.3 \mathrm{GHz}$ and $300 \mathrm{GHz}$. Microwaves are widely used in many civilian uses as communications, navigation, instrumentation, and power (as in microwave ovens). They are used also in military purposes for remote sensing as in radar, and guidance systems [1]. There are many produced problems accompanying this heavy use of the microwave radiation as the increase in electromagnetic pollution, leak of secret information caused by a leakage of electromagnetic wave, and electromagnetic interference which can cause severe interruption of electronically controlled systems, where it can cause device malfunctions, generate false images, increase clutter on radar and reduce performance because of system-to-system coupling. Consequently, microwave absorbers are of great interest for shielding the microwave sources. Microwave absorbers also can be used in stealth for the radar systems by minimizing the electromagnetic reflection from objects which have metallic structure such as aircrafts, ships, and automobiles [2-4].

To achieve good absorption performance and wideband width absorption of a material, the complex permittivity $\left(\varepsilon=\varepsilon^{n}-j \varepsilon^{m}\right)$ and permeability $\left(\mu=\mu^{s}-j \mu^{n}\right)$ should be considered. Accordingly, composite materials consisting of magnetic media and electronic media with high magnetic and electric losses (which are known as magneto-electric composites) are widely used as electromagnetic wave absorber in the recent years. Ferrite materials exhibit various electrical and magnetic properties. The NiZn-ferrites are found to be the most versatile technological materials especially suited to high frequency applications on account of their high resistivity. However, NiZn-ferrite is generally used as magnetic materials and has poor dielectric loss [5-8]. Conducting polymers have attracted considerable attention for their potential applications in the microwave absorption field. Among the conducting polymers, polyaniline (PANI) has been extensively studied in the last two decades due to its unique electrochemical and physicochemical behavior, good environment stability, low density, controllable conductivity, and relative ease of preparation [5,9].

Covering the ferrite nanoparticles by conductive polymer (as in the core-shell composites) can enhance compatibility with organic ingredients, reduce susceptibility to leaching, and probably avoid aggregation [10]. The additional interfaces occurred in the core-shell composites introduce more polarization charges on the surface of the particles. This could improve the dielectric and joule-heating losses due to finite conductivity of the ferrite core $[8]$.

Microwave absorbing material (in sheet form) should present tiny thickness, flexibility, low density, light weight, and wide bandwidth absorption. This can be achieved by loading the electromagnetic powders in an appropriate binder [12].

In this work, preparation of a composite structural PANI/NiZn-ferrite is presented. Firstly, NiZn-ferrite was prepared by the coprecipitation technique which is known as a simple route to prepare fine, nano-crystallized, high-purity and homogeneous powders of single or multicomponent oxides [11]. The fine of NiZn-ferrite particulates product was then shelled by PANI. Sheets of the absorbing material were produced by loading the final core-shell powder into epoxy matrix.

The influence of the PANI content with respect to the electromagnetic properties of PANI/NiZn ferrite composites has been investigated. The origin of their electromagnetic 
properties is also discussed on the basis of the structural characterization; including Fourier transform infrared (FTIR), X-ray diffraction (XRD), and transmission electron microscopy (TEM). The four probe method technique has been used for investigating the electrical conductivity. Microwave reflection loss of the samples reinforced epoxy resin composites was tested at 8-18 GHz microwave frequencies using the free space method, which is known to be a suitable technique for measuring those of anisotropic and inhomogeneous material composites [13].

\section{Experimental Work}

\subsection{Materials}

Ferric nitrate $\left(\mathrm{Fe}\left(\mathrm{NO}_{3}\right)_{3} .9 \mathrm{H}_{2} \mathrm{O}\right.$, Alpha, $\left.99 \%\right)$, nickel chloride $\left(\mathrm{NiCl}_{2} \cdot 6 \mathrm{H}_{2} \mathrm{O}\right.$, Winlab,98\%), zinc chloride $\left(\mathrm{ZnCl}_{2}\right.$, Molychem, $\left.95 \%\right)$, and sodium hydroxide $(\mathrm{NaOH}, \mathrm{El}$ nasr pharmachemical Co., 96\%) were used in the preparation of NiZn-ferrite. Re-distilled aniline (El nasr pharmachemical Co.), and hydrochloric acid ( $\mathrm{HCl}, \mathrm{BDH}$ chemicals Ltd, 36\%), ammonium persulfate (APS, $\left(\mathrm{NH}_{4}\right)_{2} \mathrm{~S}_{2} \mathrm{O}_{8}$, Alpha, 99\%) were used for the preparation of PANI, epoxy (ISIPAC company, Egypt).

\subsection{Preparation epoxy-PANI/NiZn-ferrite composites}

\subsubsection{Preparation of NiZn-ferrite nano powders}

The NiZn-ferrite magnetic particles were prepared by the chemical coprecipitation method. A stoichiometric ratios of metal salts which give a composition of $\mathrm{Ni}_{0.5} \mathrm{Zn}_{0.5} \mathrm{Fe}_{2} \mathrm{O}_{4}(35.0378 \mathrm{~g}$ $\mathrm{Fe}\left(\mathrm{NO}_{3}\right)_{3} \cdot 9 \mathrm{H}_{2} \mathrm{O}, 5.1014 \mathrm{~g} \mathrm{NiCl}_{2} \cdot 6 \mathrm{H}_{2} \mathrm{O}$ and $\left.3.0172 \mathrm{~g} \mathrm{ZnCl}_{2}\right)$ are dissolved in $250 \mathrm{ml}$ distilled water and was introduced drop-by-drop, with vigorous stirring, into $\mathrm{NaOH}$-solution (2L reaction beaker containing $1150 \mathrm{ml}$ of $1 \mathrm{~N} \mathrm{NaOH}$ heated at $90{ }^{\circ} \mathrm{C}$ ), then the temperature was maintained at $90{ }^{\circ} \mathrm{C}$ for 90 minutes with continuous stirring. The obtained co-precipitate was washed with deionized water to remove impurities until attaining $\mathrm{pH}$ value of 7 . At the end, the product was washed by ethanol to avoid the formation of agglomerates. The powder was dried at $80{ }^{\circ} \mathrm{C}$ for $24 \mathrm{~h}$.

\subsubsection{Preparation of PANI/NiZn-ferrite nanocomposite}

PANI-ferrite composite was prepared by in situ polymerization in aqueous solution. Ferrite particles $(10 \mathrm{~g})$ were suspended in an appropriate volume of $\mathrm{HCl}$ solution $(1 \mathrm{M})$. The volume was adjusted to get aniline concentration of $0.55 \mathrm{M}$. the solution was then vigorously stirred at room temperature for 10 minutes in an ultrasonic basin to get well dispersed particles. The subsequent addition of aniline monomer into the dispersion was performed at ambient temperature with continuous vigorous stirring for 1 hour. The composite ratios of NiZn-ferrite and aniline in weightlvolume ratio were 1:1 (PANI/NiZn-ferrite-1), 2:1 (PANI/NiZn-ferrite-2) and 3:1 (PANI/NiZn-ferrite-3), respectively, and then the dispersion was cooled to $0{ }^{\circ} \mathrm{C}$ with stirring. An appropriate amount of acidic APS solution (APS $0.23 \mathrm{M}$ and $\mathrm{HCl} 1 \mathrm{M}$ ) was then slowly added drop-wise to the suspension mixture with a constant and vigorous stirring with ultrasonic action. The amount of the APS was adjusted so as to have aniline to APS molar ratio of 1:1.25. The polymerization was allowed to proceed over night at $0{ }^{\circ} \mathrm{C}$. The final composite was obtained by filtering and washing the suspension with deionized water and acetone. The obtained filtrate is then dispersed in acetone and dried at $80{ }^{\circ} \mathrm{C}$ for $24 \mathrm{~h}$. 


\subsubsection{Preparation epoxy-PANI/NiZn ferrite composites}

The absorbing composite materials of $10 \%$ weight ratio of powders in the epoxy polymer were prepared by adding the needed amount of composite powder (portion by portion) to epoxy resin with mixing for 15 minute followed by addition of epoxy hardener with continuous mixing for 10 minute. The epoxy polymer is consisting of resin and hardener with certain weight ratio (3:1). The produced mixture was casted in iron mold with dimensions of $180 \mathrm{~mm} \times 180 \mathrm{~mm} \times 2 \mathrm{~mm}$. sheets were then dried at normal temperature for 24 hour giving iron sheet coated with resin-PANI/NiZn-ferrite which have a thickness of about $1.5 \mathrm{~mm}$.

\subsection{Experimental techniques}

FTIR has been used to confirm the formation of polyaniline. Transmittance are tabulated against the wave number for each sample of the powders under investigation within the infrared region was carried out, using JASCO FTIR-6200 instrument after calibration and automatic adjustment. About $2.0 \mathrm{mg}$ of powder and $300.0 \mathrm{mg}$ of potassium bromide $(\mathrm{KBr})$ were mixed using an agate mortar and pressed by using hydraulic press [14].

Morphological analysis and the particle size were performed using (TEM) type JOEL-1230. The TEM also contribute in the insuring the formation of the polyaniline as a shell on the surface of the ferrite as a core. The sample prepared for measurement on TEM by suspension of powders in ethanol with stirring and ultrasonic action for 30 minutes. The EDAX associated with SEM contribute in the elemental analysis of all samples. The phase identification of the samples was performed with X-ray diffraction (X Pert PRO) with $\mathrm{Cu}-\mathrm{K} \alpha$ radiation. The effect of the PANI content on the electrical conductivity of the composites was studied by four point probe coupled with Jandel HM21 test unit.

PR-17 microwave reflectometer is portable, swept frequency reflectance analyzer used for the testing of the microwave reflectivity of surfaces. It operates in free space using transmitted signal from a transmitting antenna bounced off a measurement surface to a separate receiving antenna. The transmit and receive antennas oriented at a \pm 15 degree bi-static angle compared with the surface normal to measure relative return loss. Legs mounted onto the instrument keep the distance between the antenna and the measured surface is constant as shown in fig. (1). Percentage of absorption in the transmitted and reflected beam from the measured surface at each frequency is calculated by the associated PC computer. All samples were made 180 $\mathrm{mm} \times 180 \mathrm{~mm}$ with thickness of $2 \mathrm{~mm}$ in order to cover the metallic plate for reflectivity measurements [15].

\section{Results and Discussions}

\subsection{Ferrite preparation mechanism}

The chemical reaction processes are very complex about three major chemical reactions as follows:

$$
\begin{aligned}
& 2 \mathrm{Fe}\left(\mathrm{NO}_{3}\right)_{3} \cdot 9 \mathrm{H}_{2} \mathrm{O}+0.5 \mathrm{NiCl}_{2} \cdot 6 \mathrm{H}_{2} \mathrm{O}+0.5 \mathrm{ZnCl}_{2}+8 \mathrm{NaOH} \\
& \rightarrow 2 \mathrm{Fe}(\mathrm{OH})_{3}+0.5 \mathrm{Ni}(\mathrm{OH})_{2}+0.5 \mathrm{Zn}(\mathrm{OH})_{2}+6 \mathrm{NaNO}_{3}+2 \mathrm{NaCl} \\
& 2 \mathrm{Fe}(\mathrm{OH})_{3}+0.5 \mathrm{Ni}(\mathrm{OH})_{2}+0.5 \mathrm{Zn}(\mathrm{OH})_{2} \stackrel{\Delta}{\rightarrow} \mathrm{Fe}_{2} \mathrm{O}_{3}+0.5 \mathrm{NiO}+0.5 \mathrm{ZnO}+4 \mathrm{H}_{2} \mathrm{O} \\
& \mathrm{Fe}_{2} \mathrm{O}_{3}+0.5 \mathrm{NiO}+0.5 \mathrm{ZnO} \rightarrow \mathrm{Ni}_{0.5} \mathrm{Zn}_{0.5} \mathrm{Fe}_{2} \mathrm{O}_{4}
\end{aligned}
$$




\subsection{Polymerization mechanism}

When the ferrite particles is suspended in hydrochloric acid, it is expected that the $\mathrm{Cl}^{-}$ion will attracted to the surface of the ferrite powders and form a shell on the ferrite core to compensate the positively charged ferrite surface produced due to the acidic medium. Aniline monomers, when added to the acidic medium, convert into cationic anilinium ions. The electrostatic interactions appear between adsorbed anions on the ferrite surface and the cationic anilinium ions and then aniline monomers electro-statically complexes to the ferrite surface and polymerize by APS as an oxidizing agent [9]. To minimize the presence of residual aniline and to obtain the best yield of PANI, the stoichiometric peroxydisulfate/aniline ratio 1.25 is recommended [19].
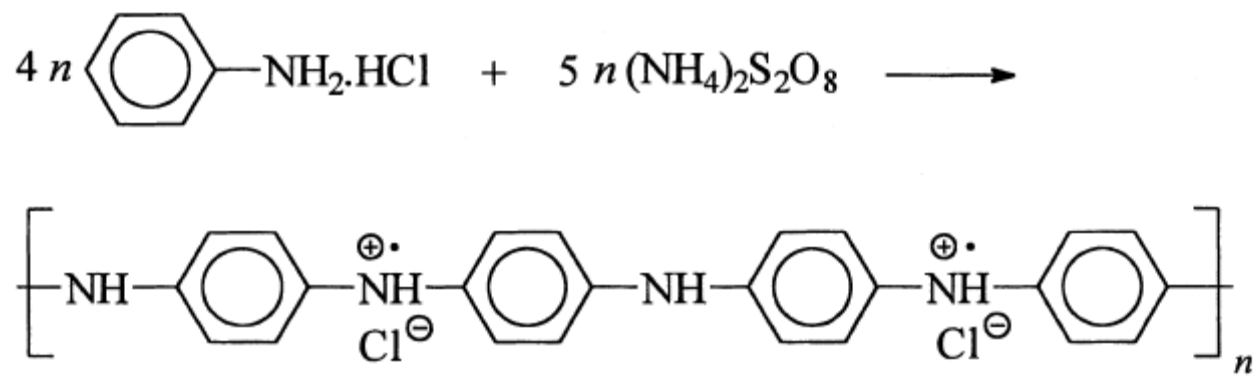

$$
+2 n \mathrm{HCl}+5 n \mathrm{H}_{2} \mathrm{SO}_{4}+5 n\left(\mathrm{NH}_{4}\right)_{2} \mathrm{SO}_{4}
$$

\subsection{Structural and morphological characterization}

The FTIR spectra of the NiZn ferrite, the composites PANI/NiZn-ferrite samples, and FTIR spectra of NiZn-ferrite and PANI from literature are shown in Fig. $(2,3,4)$. The band at 580 (1) corresponds to intrinsic stretching vibrations of the $\mathrm{Zn}^{2+}$ ion at the tetrahedral site $\left(\mathrm{Zn}^{2+} \leftrightarrow \mathrm{O}^{2-}\right)$ in the crystal lattice, while $400 \mathrm{~cm}^{-1}$ (2) is assigned to octahedral-Fe $\mathrm{Fe}^{3+}$ stretching $\left(\mathrm{Fe}^{3+} \leftrightarrow \mathrm{O}^{2-}\right)$ the characteristic band of NiZn ferrite, in addition, the absorption at $3406 \mathrm{~cm}^{-1}$ was ascribed to the stretching modes and $\mathrm{H}-\mathrm{O}-\mathrm{H}$ bending vibration of the free or absorbed water [17, 7].

With an increase in the PANI content, the intensity of the characteristic NiZn ferrite peaks decreased distinctively and the bands corresponding to NiZn ferrite shifted.

The characteristic absorption bands of PANI are (3) and (4) ( $\mathrm{C}=\mathrm{N}$ and $\mathrm{C}=\mathrm{C}$ stretching), (5) ( $\mathrm{N}-\mathrm{H}$ bending), (6) (a vibration mode of $\mathrm{N}=\mathrm{Q}=\mathrm{N}$ ) and (7) (out-of plane stretching vibration of $\mathrm{C}-\mathrm{H})$, where $\mathrm{Q}$ is quinone ring $[4,17]$.

It is clearly observed that the increase in the PANI content in the composites causes increasing in the intensity of the bands corresponding to PANI characteristics distinctively. Also, the bands characteristic for the NiZn-ferrite were greatly diminished due to the decreasing the probability of the interaction of the ferrite particles with the incident radiation during IR measurements caused by the coating of ferrite nanoparticles by the polymer. The incorporation of ferrites in the nanocomposites leads to a shift of some FTIR bands of the polymer and the ferrite in the composite when compared to PANI alone or ferrite alone [20]. 
Fig. 5 shows the XRD patterns of the NiZn ferrite particles, and the PANI/NiZn-ferrite composites. The analysis revealed a match with PDF \# 08-0234 in the International Centre for Diffraction Data, Newtown Square indicating the nickel zinc ferrite structure [16], matching with the reference pattern carried out by Match! Program (Copyright 2003-2011 CRYSTAL IMPACT, Bonn, Germany) shows the production of the spinel phase with high overall agreement between the entry database and the sample diffraction pattern.

The main diffraction peaks for PANI are with amorphous nature at about $2 \theta=20.3^{\circ}$ and $25.1^{\circ}$ [4], The XRD patterns for the composites show the presence of the PANI peaks in the positions of approximately 20.9 and $25.3^{\circ}$. It is observed that as the weight percent of the PANI increases in the NiZn ferrite/PANI composites, the intensity of the peaks corresponding to the NiZn-ferrite phase decreases and for the PANI increases. These results may indicate that the continuous over-layer of PANI over the surface of the NiZn ferrite powders has been produced.

Both the pure NiZn ferrite and composite powders are a single-phase NiZn-ferrite with a spinel structure. Therefore, the average crystallite size of the NiZn-ferrite particles could be estimated with the Scherrer's formula, $D=k \lambda / \beta \cos \theta$, where $D$ is the crystallite size $(n m), \lambda$ is the radiation wavelength $(0.154056 \mathrm{~nm}$ for $\mathrm{Cu}-\mathrm{K \alpha}), \beta$ is the bandwidth at half-height, and $\theta$ is the diffraction peak angle, $\mathrm{k}$ is the shape factor and is taken as 0.9. The main reflecting peak at $2 \theta=35.4^{\circ}$ which is the characteristic peak of NiZn-ferrite was chosen for calculating the average crystallite sizes of NiZn-ferrite [7], The calculated crystallite sizes of the NiZn-ferrite particles range from 30 to $35 \mathrm{~nm}$.

The morphology, particle size of NiZn-ferrite and PANI/NiZn ferrite composites were determined by TEM. In Fig. 6, it is obvious that the NiZn-ferrite particles are approximately spherical. TEM image for the composite indicates that the ferrite particles are embedded in the polyaniline matrix forming the core-shell structure. The black core is ferrite particle with the diameter in the range of $70-100 \mathrm{~nm}$, and the light colored shell is PANI in the nanocomposites, due to the different electron penetrability. The surface composition of the NZ-ferrite and the PANI/NiZn-ferrite-1 composite was determined by the EDAX analysis carried out by SEM as shown in Fig. 7. The weight ratio of the elements present in the prepared NiZn-ferrite ( $\mathrm{Ni}: \mathrm{Zn}$ : Fe: $\mathrm{O}$ ) indicated the right elemental composition of the prepared ferrite sample. It is observed the appearance of carbon and chlorine peaks in the composite samples with intensity indicate the PANI contents in them and the intensity of the characteristic peaks of the NiZn-ferrite are decreased by increasing the PANI content in the composite. The presence of sulfur peaks in the spectrum of composite is due to the impurities produced from APS was used in the preparation.

\subsection{The electrical conductivity}

Table 1 summarizes the effect of the PANI content on the electrical conductivity of the PANI/NiZn ferrite composites. The electrical conductivity of the PANI/NiZn ferrite composite was increased with increasing the PANI content in the composite. 


\subsection{Reflectivity measurements}

Fig. 8 shows the frequency dependence of the reflection loss of the epoxy-NiZn ferrite composite and epoxy-PANI/NiZn-ferrite composites in the frequency range of 8-18 GHz. It can be seen the enhancement in the microwave absorption at $8-18 \mathrm{GHz}$ occurred by the presence of PANI in the composite. It is observed that by increasing the PANI content in the composite the absorption in the x-band increases which attain -2.36946 in the specimen PANI/NiZn-ferrite-3. The best enhancement in the absorption was in the specimen PANI/NiZn-ferrite-1 with average reflection loss $-3.907 \mathrm{~dB}$. The maximum reflection loss frequency changed from $17.3 \mathrm{GHz}$ in the specimen PANI/NiZn-ferrite- 1 to $8.3 \mathrm{GHz}$ in the specimen PANI/NiZn-ferrite-3. The results indicate that the composites exhibit good absorption performances over a broader band range than in case of using NiZn-ferrite only and the maximum reflection loss frequency can be determined by controlling aniline to NiZnferrite ratio. .

\section{Conclusions}

The structural PANI/NiZn-ferrite composites exhibiting electromagnetic properties were successfully synthesized by in situ polymerization method. FTIR, XRD, and TEM studies have established formation of spinel structure of NiZn-ferrite and the PANI/NiZn-ferrite coreshell composites. The electrical conductivity property of the NiZn-ferrite is enhanced by covering its surface with the conductive and increased with the increasing PANI content. Clearly demonstrated is that the intensity and frequency of the reflection loss maximal for the composite also depend on the PANI content. The best enhancement in the absorption was in the specimen PANI/NiZn-ferrite-1 with average reflection loss $-3.907 \mathrm{~dB}$ and maximum reflection loss $-8.698 \mathrm{~dB}$ at $17.3 \mathrm{GHz}$.

\section{References}

[1] Microwave - Wikipedia, the free encyclopedia.

[2] Ali Ghasemi. et al., Magnetic and microwave absorption properties of $\mathrm{BaFe}_{12-\mathrm{x}}$ $\left(\mathrm{Mn}_{0.5} \mathrm{Cu}_{0.5} \mathrm{Zr}\right)_{\mathrm{x} / 2} \mathrm{O}_{19}$ synthesized by sol-gel processing, J. of Magnetism and Magnetic Materials 316 (2007) e105-e108.

[3] Y.B. Feng et al., Absorbing properties and structural design of microwave absorbers based on carbonyl iron and barium ferrite, J. of Magnetism and Magnetic Materials 318 (2007) 8-13.

[4] T.H. Ting. et al., Synthesis and microwave absorption characteristics of polyaniline/NiZn ferrite composites in 2-40GHz, Materials Chemistry and Physics 126 (2011) 364-368.

[5] Xin Tang et al., Surface modification of M-Ba-ferrite powders by polyaniline: Towards improving microwave electromagnetic response, Applied Surface Science 255 (2009) 9381-9385

[6] Yeon Hwang, Microwave absorbing properties of NiZn-ferrite synthesized from waste iron oxide catalyst, Materials Letters 60 (2006) 3277-3280.

[7] K.H. Wu et al., Electromagnetic and microwave absorbing properties of $\mathrm{Ni}_{0.5} \mathrm{Zn}_{0.5} \mathrm{Fe}_{2} \mathrm{O}_{4} /$ bamboo charcoal core-shell nanocomposites, Composites Science and Technology 68 (2008) 132-139. 
[8] Xin Tang et al., Preparation and electromagnetic wave absorption properties of Fedoped zinc oxide coated barium ferrite composites, Materials Science and Engineering B 139 (2007) 119-123.

[9] Jing Jiang et al., Polyaniline-LiNi ferrite core-shell composite: Preparation, characterization and properties, Materials Science and Engineering A 456 (2007) 300304.

[10] Liangchao Li et al., $\mathrm{Zn}_{0.6} \mathrm{Cu}_{0.4} \mathrm{Cr}_{0.5} \mathrm{Fe}_{1.46} \mathrm{Sm}_{0.04} \mathrm{O}_{4}$ ferrite and its nanocomposites with polyaniline and polypyrrole: Preparation and electromagnetic properties, Synthetic Metals Xxx (2009) xxx-Xxx.

[11] M.M. Rashad et al., Synthesis and characterization of nano-sized nickel ferrites from fly ash for catalytic oxidation of CO, Materials Chemistry and Physics 94 (2005) 365370.

[12] J.C. Aphesteguy et al., Microwave absorption behavior of a polyaniline magnetic composite in the X-band, Physica B (2012)

[13] C.C. Yang et al., Synthesis, infrared and microwave absorbing properties of $\left(\mathrm{BaFe}_{12} \mathrm{O}_{19}+\mathrm{BaTiO}_{3}\right) /$ polyaniline composite, J. of Magnetism and Magnetic Materials 323 (2011) 933-938.

[14] Kenneth A. R. et al., Contemporary Instrumental Analysis, Prentice Hall (2000).

[15] Millimeter Wave Technology Inc., "Swept Frequency Reflectometer PR-17", Device Catalog, Georgia, (2004).

[16] Shannon A. Morrison et al., Magnetic and structural properties of nickel zinc ferrite nanoparticles synthesized at room temperature, J. of Applied Physics, no.11,vol. 95 (2004) $6392-6395$.

[17] Xing-Rong Zeng et al., structure and properties of chemically reduced polyanilines, pII S0032-3861(1997) 00381-9

[18] Mona Mouallem-Bahout et al., Synthesis and characterization of $\mathrm{Zn}_{1-x} \mathrm{Ni}_{\mathrm{x}} \mathrm{Fe}_{2} \mathrm{O}_{4}$ spinels prepared by a citrate precursor, J. of Solid State Chemistry 178 (2005) 1080-1086.

[19] J. STEJSKAL, Polyaniline preparation of a conducting polymer, Pure Appl. Chem. 745 (2002) 857-867.

[20] Boris Renteria Beleño, Preparation and Characterization of Polyaniline-Based Magnetic Nanocomposites for EMI Shielding Applications, Master of Science (2007). 


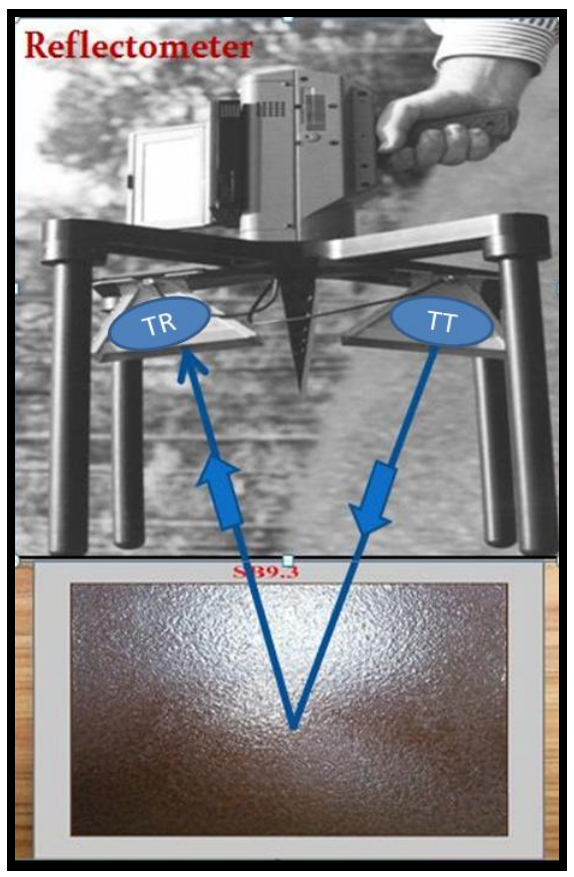

Fig. (1) Swept frequency reflectometer model PR-17 


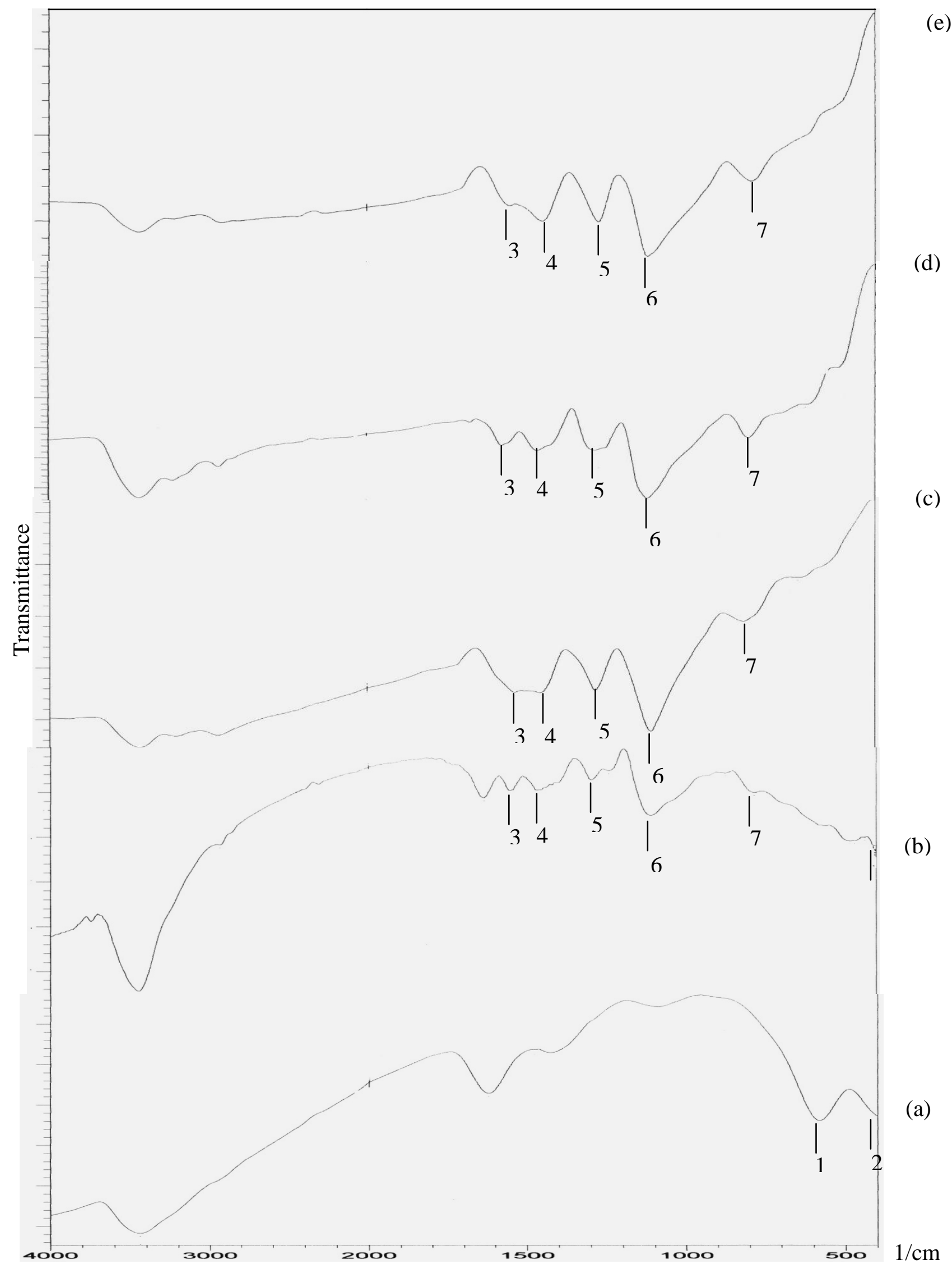

Fig. (2) FTIR spectra of (a) NiZn-ferrite, (b) PANI/NiZn-ferrite-1, (c) PANI/NiZn-ferrite-2, (d) PANI/NiZn-ferrite-3 composites and (e) PANI. 


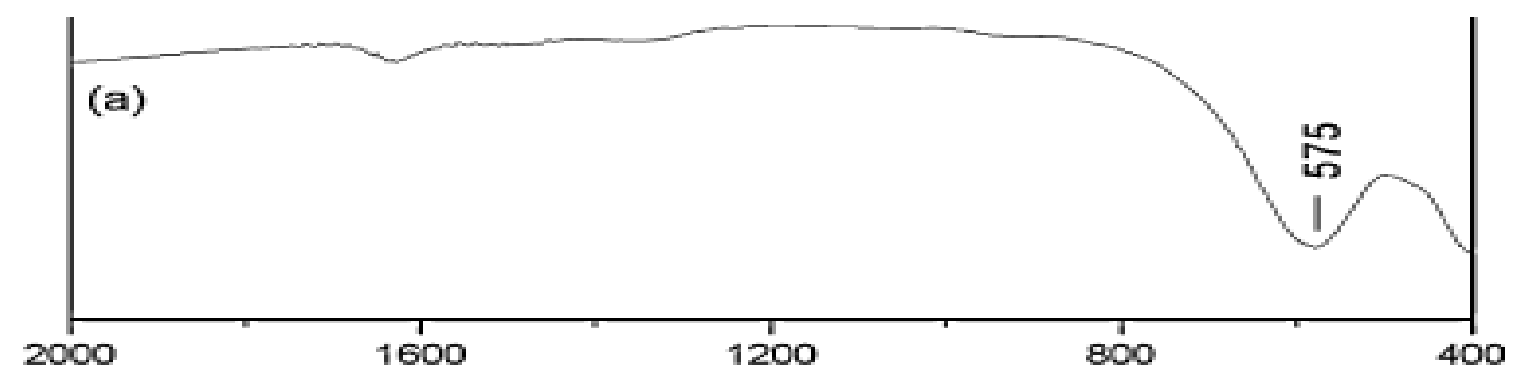

Fig. (3) Comparison between (a) a PANI standard FTIR spectra and (b) the prepared PANI sample FTIR spectra.

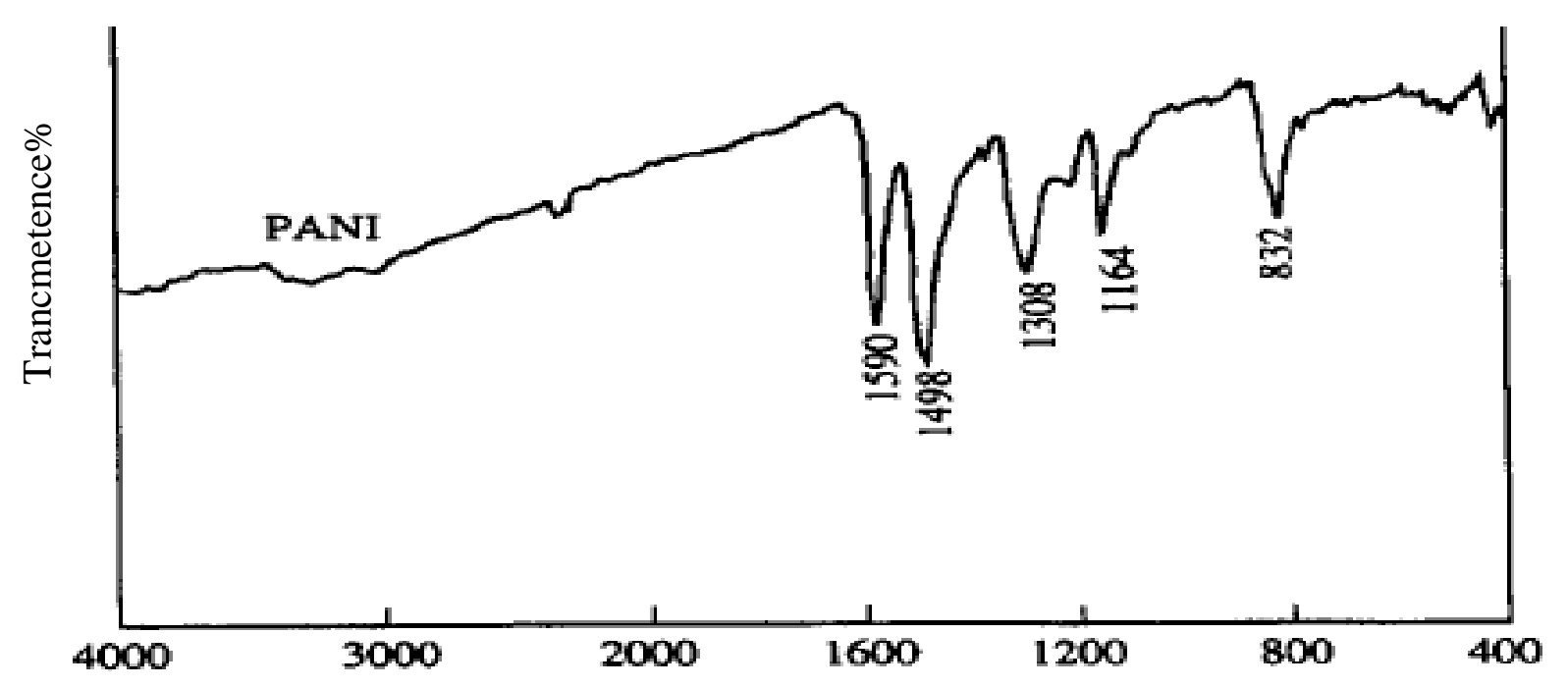

Fig. (4) Comparison between (a) a standard FTIR spectra and (b) the prepared NiZn(b) ferrite sample FTIR spectra. 

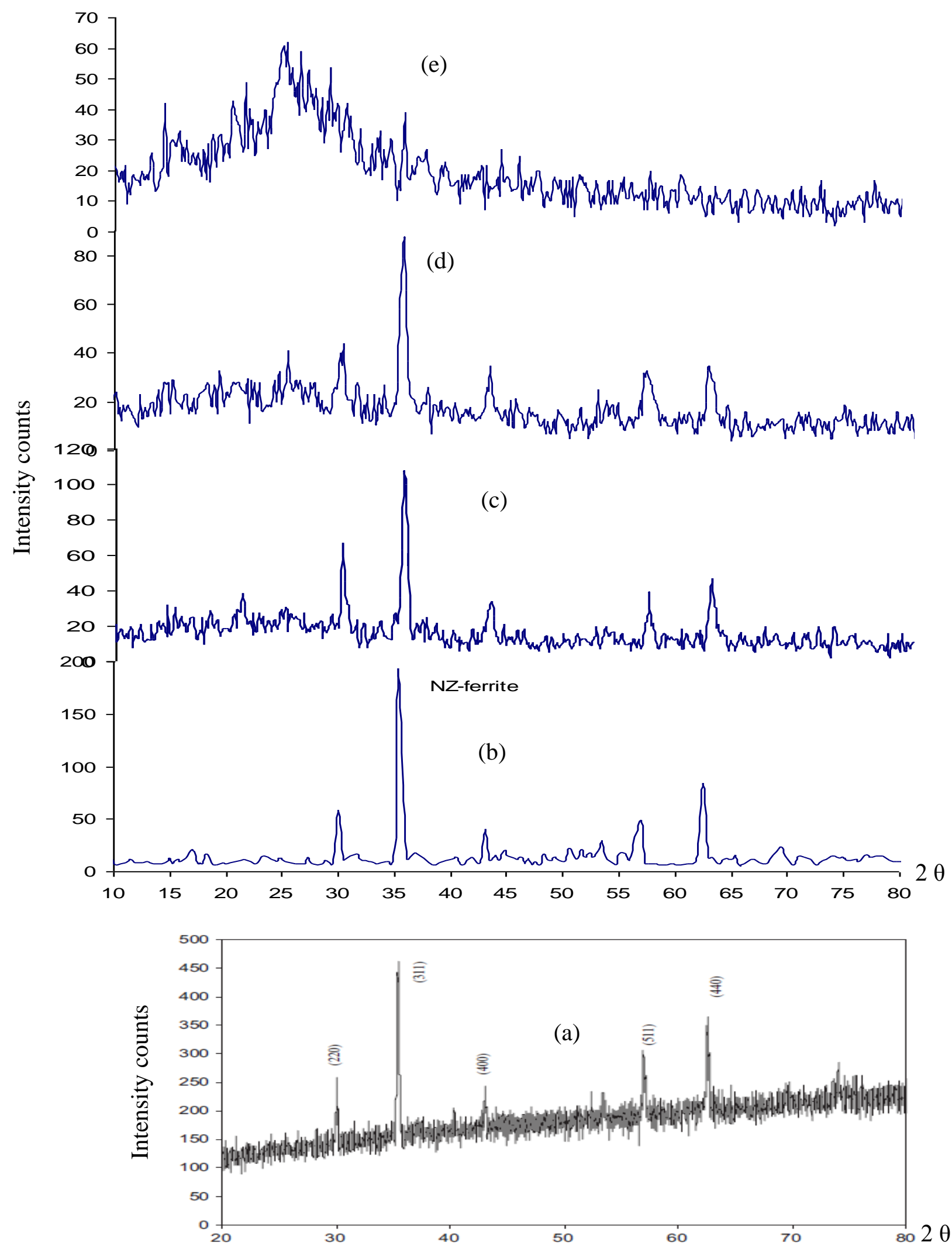

Fig. (5) XRD spectra of (a) NiZn ferrite standard (b) NiZn ferrite sample, (c) PANI/NiZn ferrite-1, (d) PANI/NiZn ferrite-2, and (e) PANI/NiZn ferrite-3 composites. 


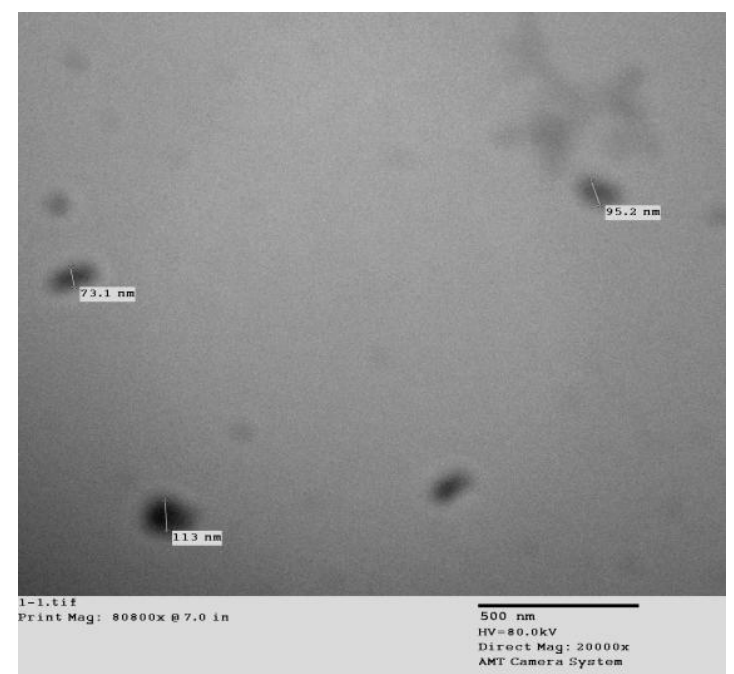

Fig. (6) TEM photograph for PANI/NiZn ferrite-1 composite.

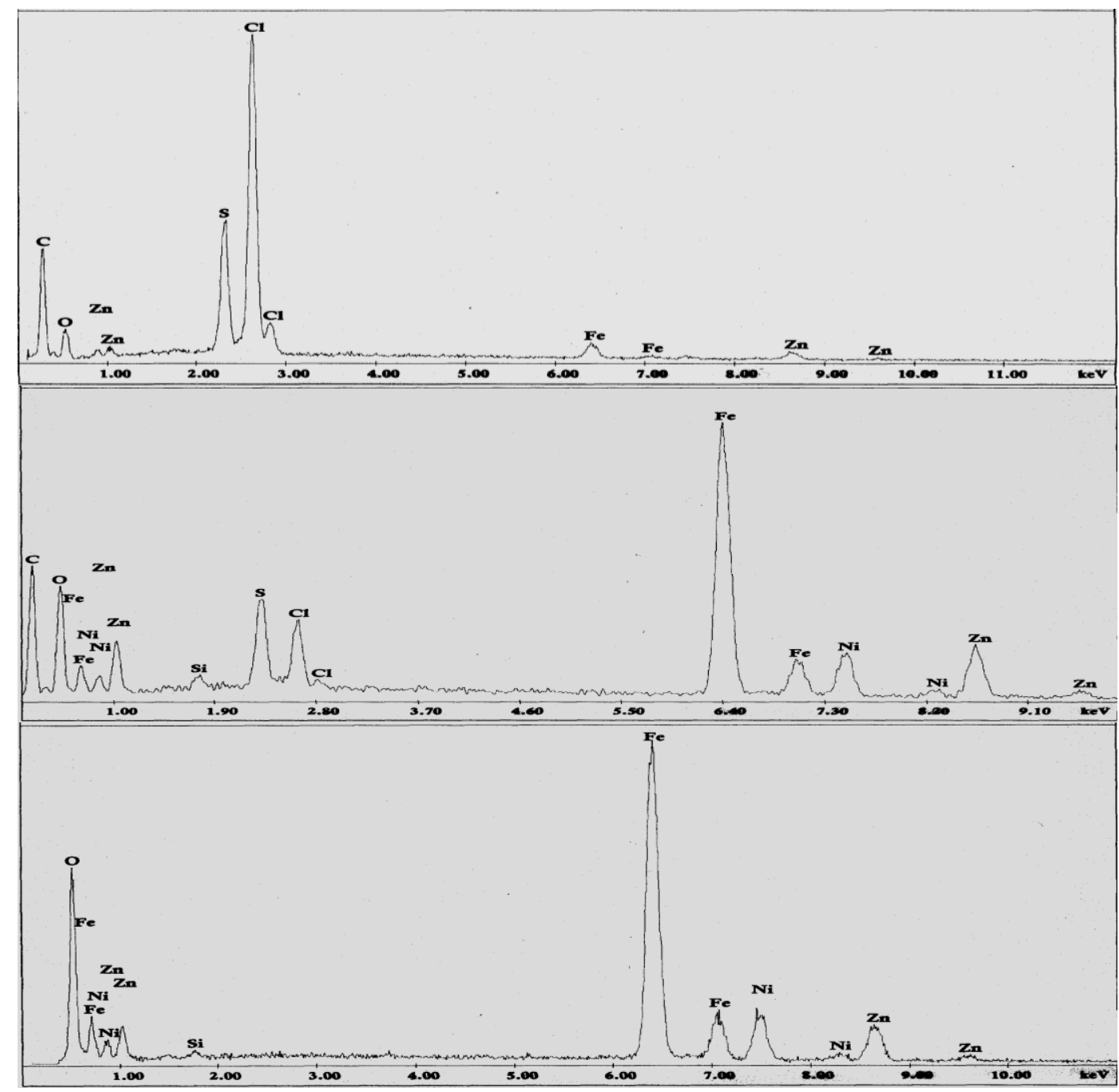

(c)

(b)

(a)

Fig. (7) EDAX spectrum of the (a) NiZn ferrite, (b) PANI/NiZn ferrite-1, (c) PANI/NiZn ferrite-3 
(e)

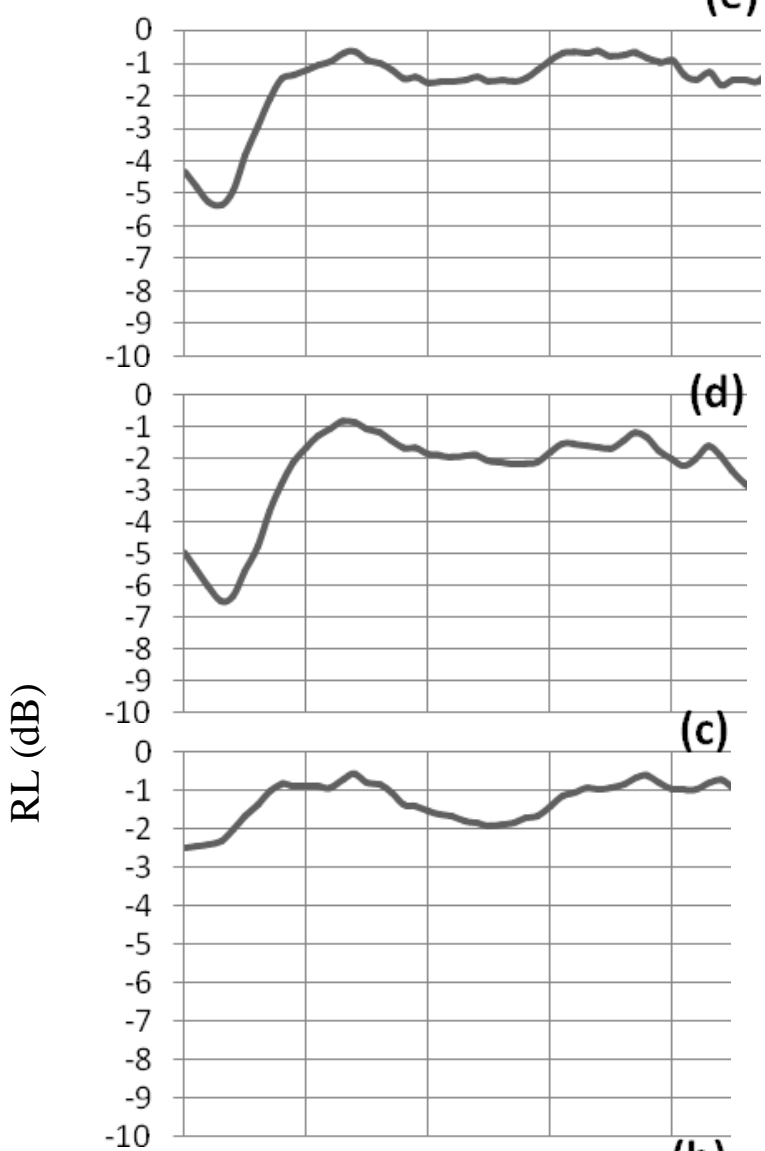

(b)

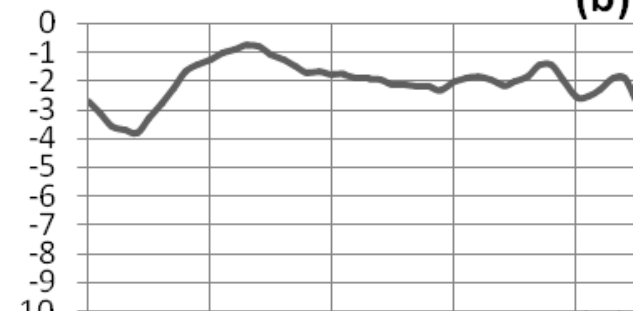

(a)

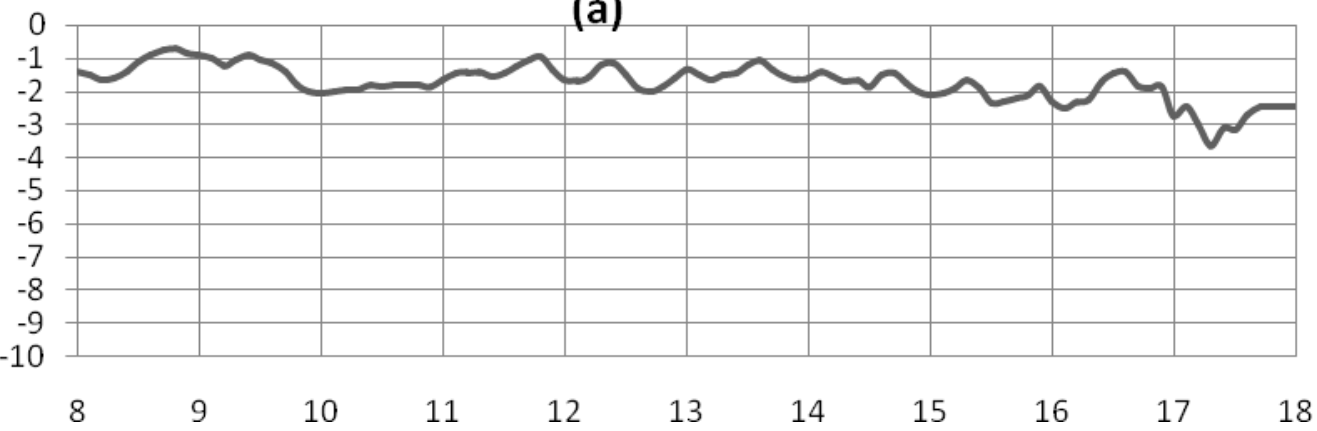

Frequency $(\mathrm{GHz})$

Fig. (8) Microwave absorbing properties of $10 \%$ weight percent of powder in epoxy-(a) NiZn ferrite, (b) PANI/NiZn ferrite-1, (c) PANI/NiZn ferrite-2, (d) PANI/NiZn ferrite-3 composites and (e) PANI in 8-18 GHz. 
Table (1) Conductive characteristics of PANI and PANI/NiZn-ferrite composites.

\begin{tabular}{|c|c|c|c|c|c|}
\hline Sample & NiZn-ferrite & $\begin{array}{c}\text { PANI/NiZn- } \\
\text { ferrite-1 }\end{array}$ & $\begin{array}{c}\text { PANI/NiZn- } \\
\text { ferrite-2 }\end{array}$ & $\begin{array}{c}\text { PANI/NiZn- } \\
\text { ferrite-3 }\end{array}$ & PANI \\
\hline $\begin{array}{c}\text { Conductivity } \\
\text { (S\cm) }\end{array}$ & Out of range & $3.96 \times 10^{-3}$ & $4.16 \times 10^{-3}$ & $4.56 \times 10^{-3}$ & $5.45 \times 10^{-3}$ \\
\hline
\end{tabular}

